\title{
Development of Insulin Resistance in Normal Dogs Following Alloxan-Induced Insulin Deficiency
}

\author{
G. M. Reaven ${ }^{1}$, W. S. Sageman, and R. S. Swenson \\ Department of Medicine, Stanford University School of Medicine and Veterans Administration Hospital, Palo Alto, California, USA
}

\begin{abstract}
Summary. Insulin resistance was measured in 16 normal dogs by a method involving the continuous intravenous infusion of epinephrine, propranolol, glucose and insulin. With this approach, endogenous insulin secretion is inhibited, similar steady state levels of exogenous insulin are achieved in all dogs, and the resultant steady state plasma glucose level provides a direct estimate of the ability of insulin to dispose of the infused glucose load. Thus, the higher the steady state plasma glucose level, the more the insulin resistance. Different amounts of alloxan were then administered to these dogs in order to produce insulin deficiency of varying degrees. Insulin resistance was then measured again in each dog. The results indicated that insulin resistance did not develop in dogs with only a moderate degree of insulin deficiency (fasting plasma glucose levels $<150 \mathrm{mg}$ / $100 \mathrm{ml}$ ). On the other hand, a significant degree of insulin resistance developed in dogs with severe insulin deficiency (fasting plasma glucose > $150 \mathrm{mg} / 100 \mathrm{ml}$ ). Furthermore, the insulin resistance that developed in dogs with severe insulin deficiency could be returned to normal with insulin replacement for one week. These results indicate that insulin resistance can occur as a secondary manifestation of insulin deficiency.
\end{abstract}

Key words: Diabetes, insulin deficiency, hyperglycaemia, insulin resistance, insulin treatment, alloxan.

In the past several years our laboratory has published several articles documenting the existence of insulin resistance in patients with nonketotic dia-

\footnotetext{
1 Dr. Reaven is a Medical Investigator, Veterans Administration
}

betes mellitus [1-6]. In the case of many patients with chemical diabetes the insulin resistance occurs in association with an insulin response to oral glucose which is equal to or greater than that seen in normal subjects, and we have suggested that the glucose intolerance in these patients is secondary to the insulin resistance [7]. On the other hand, patients with fasting hyperglycaemia seem to have both insulin resistance and a decrease in their insulin response [7]. In this case, the hyperglycaemia could be due to either abnormality. We have previously suggested that it is the defect in insulin secretion that is primarily responsible for the hyperglycaemia in these patients, and that the insulin resistance develops as a consequence of the insulin deficiency [7]. In an effort to provide support for this hypothesis we have carried out the present study in which we have quantified insulin resistance in dogs before and after the development of varying degrees of alloxaninduced insulin deficiency.

\section{Materials and Methods}

\section{Experimental Protocol}

Two estimates of glucose tolerance were carried out in 16 nonobese, healthy mongrel dogs before the induction of experimental diabetes. The first consisted of a standard intravenous glucose tolerance test (IVGTT), the second of a direct determination of insulin resistance. Insulin resistance was also determined after insulin deficiency had been induced by injecting each dog with alloxan monohydrate, varying in amount from $30-60 \mathrm{mg}$ per $\mathrm{kg}$ body weight. Blood was obtained for determination of fasting glucose levels every other day for the next week to evaluate the severity of the degree of diabetes. Dogs 
whose fasting plasma glucose levels were consistently above $125 \mathrm{mg} / 100 \mathrm{ml}$ were considered to be diabetic and were not subjected to another IVGTT. Dogs whose fasting plasma glucose levels were less than $125 \mathrm{mg} / 100 \mathrm{ml}$ underwent a second IVGTT. The fractional disappearance of glucose from plasma ( $\mathrm{k}$ value) was determined from glucose values at 5 , 15,30 , and 60 minutes and expressed as a percent. Dogs whose $\mathrm{k}$ value fell at least $50 \%$ were considered to be diabetic. Dogs without either fasting hyperglycaemia or a decrease in $\mathrm{k}$ value were not studied further. No dogs were ketonaemic as defined by the use of Acetest tablets (Ames Co.).

\section{Evaluation of Glucose Metabolism}

Measurement of IVGTT: Dogs were anaesthetized with sodium pentobarbital $(30 \mathrm{mg} / \mathrm{kg}$ body weight $)$ and $0.5 \mathrm{~g}$ of glucose $/ \mathrm{kg}$ body weight was administered by rapid intravenous injection. Venous blood was drawn for measurement of plasma glucose into tubes containing sodium fluoride and potassium oxalate before glucose was given and 5, 15, 30 and 60 minutes after glucose administration. Plasma was separated immediately by centrifugation and stored frozen.

Insulin Resistance: These studies were carried out with a method previously described and validated [3], in which experimental subjects receive a constant intravenous infusion of epinephrine, propranolol, glucose and crystalline insulin. By this procedure endogenous insulin secretion is inhibited and similar circulating levels of exogenous insulin are produced in each animal. Under these conditions, the height of the steady state plasma glucose concentration which results from the continuous glucose infusion is a direct measure of the ability of exogenous insulin to promote disposition of the infused glucose load. Each dog was given a loading dose of propranolol $(0.07 \mathrm{mg} / \mathrm{kg})$ and then received an infusion of glucose $(18 \mathrm{mg} / \mathrm{kg}$ of body weight $/ \mathrm{min})$, epinephrine $(0.08 \mu \mathrm{g} / \mathrm{kg} / \mathrm{min})$, propranolol $(1 \mu \mathrm{g} /$ $\mathrm{kg} / \mathrm{min})$, and porcine insulin $(24 \mathrm{mU} / \mathrm{min})$ into the cephalic vein. Blood was drawn from a catheter placed in the saphenous vein before and every 10 minutes (starting at 90 minutes) after the infusion was begun. The mean steady state plasma glucose and insulin responses were determined from five samples drawn 90,100,110, 120 and 130 minutes after the beginning of the infusion. Results were excluded if the coefficient of variation of the mean steady state value exceeded $10 \%$.

\section{Analytical Methods}

Plasma glucose was measured by a glucose oxidase oxygen-rate method [8], and immunoreactive insulin by the technique of Desbuquois and Aurbach [9]. Glucose and insulin from studies before and after alloxan were measured on the same day in each dog to minimize technical variation.

\section{Results}

Fasting plasma glucose levels before and after the induction of alloxan-induced insulin deficiency are seen in Figure 1. Dogs have been divided into those with moderate (fasting glucose level $<150 \mathrm{mg}$ / $100 \mathrm{ml}$ ), and severe post-alloxan diabetes (fasting glucose levels $>150 \mathrm{mg} / 100 \mathrm{ml}$ ).

Figure 2 illustrates the mean ( \pm SEM) steady state plasma glucose and insulin levels that were attained during the infusion before and after the induction of alloxan-induced insulin deficiency. The steady state plasma insulin levels are similar before and after alloxan in both groups of dogs. Steady state plasma glucose levels increased in dogs with moderate diabetes from a mean ( \pm SEM) of $179 \pm$ $13 \mathrm{mg} / 100 \mathrm{ml}$ before to $220 \pm 15 \mathrm{mg} / 100 \mathrm{ml}$ after alloxan $(\mathrm{p}<0.05)$. The effect of insulin deficiency on insulin resistance is much more striking in dogs with severe diabetes, with the mean ( \pm SEM) steady state plasma glucose level rising from $160 \pm$ $23 \mathrm{mg} / 100 \mathrm{ml}$ to $625 \pm 47 \mathrm{mg} / 100 \mathrm{ml}$ after the induction of insulin deficiency $(\mathrm{p}<0.001)$.

The above results indicate that steady state plasma glucose levels are higher as a result of insulin deficiency, and the more severe the fasting hyperglycaemia, the greater the rise in these levels. However, each dog is starting from an increased fasting plasma glucose level after they have become diabetic. Thus, steady state plasma glucose levels may be higher in dogs with severe fasting hyperglycaemia because of their higher fasting glucose levels, without this necessarily meaning that they are more insulin resistant than dogs with moderate diabetes and lower fasting and steady state plasma glucose levels. In order to deal with this issue we also analyzed our data by subtracting the fasting level from the steady state plasma glucose level for each dog, and determining the incremental changes in glucose level above fasting that obtained during each infusion. These results are seen in Figure 3 and indicate that the incremental rise in glucose level is similar before and after alloxan in dogs with moderate diabetes. In contrast, the incremental rise after alloxan is still much greater in dogs with severe diabetes, indicating that insulin resistance does exist in this situation. 


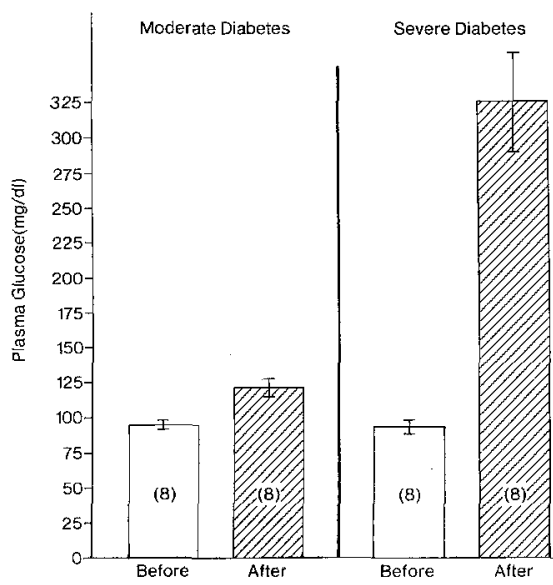

1

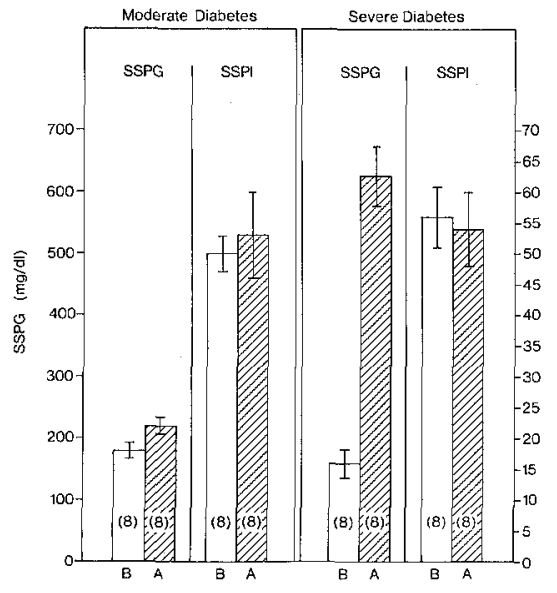

2

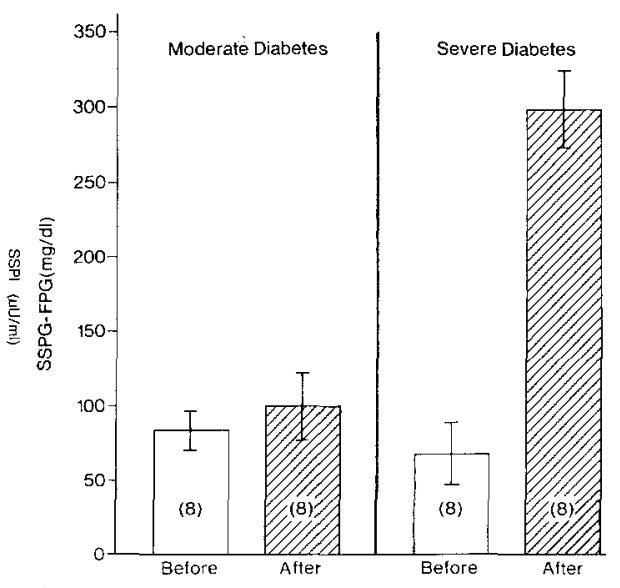

3

Fig. 1. Mean ( \pm SEM) fasting plasma glucose levels of dogs before $\Phi$ and after alloxan-induced insulin deficiency. Dogs have been divided into two groups of eight animals each: moderate diabetes (fasting glucose $<150 \mathrm{mg} / 100 \mathrm{ml}$ ) and severe diabetes (fasting glucose $>150 \mathrm{mg} / 100 \mathrm{ml}$ )

Fig. 2. Steady state plasma glucose (SSPG) and steady state plasma insulin (SSPI) levels before $\Psi$ and after alloxan-induced insulin deficiency in dogs with moderate and severe diabetes

Fig. 3. Incremental rises in steady state plasma glucose level(above fasting glucose level) before $\Psi$ and after alloxan-induced insulin deficiency in dogs with moderate and severe diabetes

Table 1. Effect of insulin replacement on insulin resistance

\begin{tabular}{|c|c|c|c|c|c|c|}
\hline & \multicolumn{3}{|c|}{ Fasting glucose level $(\mathrm{mg} / 100 \mathrm{ml})$} & \multicolumn{3}{|c|}{ Steady state glucose level $(\mathrm{mg} / 100 \mathrm{ml})$} \\
\hline & Before alloxan & After alloxan & Insulin replacement & Before alloxan & After alloxan & Insulin replacement \\
\hline $\operatorname{Dog} 1$ & 87 & 442 & 105 & 117 & 642 & 120 \\
\hline $\operatorname{Dog} 2$ & 95 & 337 & 123 & 143 & 627 & 186 \\
\hline
\end{tabular}

Two of the eight dogs with severe hyperglycaemia were treated with insulin for one week and studied on a third occasion. These results are seen in Table 1. They demonstrate in the two dogs studied that insulin therapy returns both fasting and steady state plasma glucose levels towards normal. These data indicate that the insulin resistance that develops after alloxan can be returned to normal by insulin therapy.

\section{Discussion}

The results we have presented indicate that the ability of an acute infusion of insulin to lower plasma glucose is attenuated in dogs made insulin deficient by alloxan. The observed resistance to insulin increases in parallel with severity of fasting hyperglycaemia, and normal insulin sensitivity can be restored by controlling the diabetes with exogenous insulin. Thus, it appears that insulin resistance can develop secondary to insulin deficiency. Somewhat similar results have been recently reported by Pupo et al. [10], who indicated that resistance to the acute administration of insulin could occur secondary to alloxan-induce insulin deficiency in dogs. However, in contrast to the current study, Pupo and associates were able to demonstrate insulin resistance in alloxan-diabetic dogs in the absence of severe fasting hyperglycaemia. There is no obvious reason for the discrepancy in results, and it is possible that their insulin infusion method is more sensitive than ours in documenting small differences in relative degrees of insulin resistance. Some support for this possibility can be derived from our study of five patients with moderate diabetes (fasting glucose between 125 and $187 \mathrm{mg} / 100 \mathrm{ml}$ ) secondary to chronic pancreatitis, in whom we could demonstrate normal insulin sensitivity [6]. Thus, there seems to be some question as to the severity of insulin deficiency necessary to lead to the development of insulin resistance. On the other hand, the current results, and the studies of Pupo and associates agree that insulin resistance can evolve as a secondary manifestation of insulin deficiency.

The means by which insulin deficiency can lead to insulin resistance remain to be clarified. Work from our laboratory has suggested that the insulin resistance of insulin deficient patients is secondary to the inability of insulin to inhibit hepatic glucose output and to promote the efficiency of glucose uptake [11]. The specific mechanisms responsible for this interference with insulin action could theoreti- 
cally be related to the presence of circulating insulin antagonists and/or to changes in cellular responsiveness to insulin's action. Indeed, these two phenomena could also be related, i. e., an increase in the level of a circulating insulin antagonist could lead to the development of tissue resistance to insulin. Since none of the dogs was ketonaemic, elevated levels of ketone bodies and $\mathrm{H}^{+}$do not appear responsible for the development of insulin resistance. On the other hand, elevated FFA levels could certainly contribute to the insulin resistance. Furthermore, growth hormone and glucagon are well-recognized insulin antagonists and evidence has been presented indicating that the elevated levels of these hormones that occur in insulin deficient diabetics can be returned to normal following treatment $[12,13]$. Thus, several circulating factors might be responsible for the development of the secondary insulin resistance noted in our dogs. In this regard, it has also been shown that the decrease in number of insulin receptors on circulating mononuclear leukocytes from nonketotic diabetic patients with fasting hyperglycaemia [14] can also be returned towards normal following treatment [15]. Therefore, this offers another explanation for the insulin resistance noted in alloxan diabetic dogs with severe fasting hyperglycaemia. Finally, Galton and Wilson [16] have described abnormalities in the intracellular glucose metabolism of adipocytes from adult onset diabetics. They suggested that the decrease in glucose uptake in such patients might be secondary to a decrease in phosphofructokinase levels and that this defect could be normalized following treatment of the diabetes. It is apparent that this, or other abnormalities of intracellular glucose metabolism, could also account for the development of insulin resistance.

Finally, although it is always dangerous to extrapolate from animal studies to man, the results we have presented appear to have some relevance to the pathogenesis of nonketotic diabetes in man. We have previously pointed out that two abnormalities exist in nonketotic diabetic patients with fasting hyperglycaemia: they have an insulin response to an acute glucose load which is less than normal and they are more resistant than normal to the blood glucose lowering effect of an acute insulin infusion [7]. We postulated that the insulin deficiency is the primary cause of their diabetes and the insulin resistance a secondary consequence of the insulin deficient state. The fact that insulin resistance can develop in dogs rendered severely insulin deficient by alloxan is compatible with this hypothesis. Finally, the fact that insulin resistance could not be demonstrated in dogs with only a moderate degree of hyperglycaemia suggests that insulin resistance does not necessarily develop in subjects with milder degrees of diabetes.
Acknowledgement. This work was supported in part by a grant from the National Institutes of Health, NHLI HL 08506, and from the Medical Research Service of the Veterans Administration.

\section{References}

1. Reaven, G. M., Farquhar, J.W.: Steady state plasma insulin response to continuous glucose infusion in normal and diabetic subjects. Diabetes 18, 273-279 (1969)

2. Reaven, G. M., Silvers, A., Farquhar, J. W.: Study of the relationship between plasma insulin concentration and efficiency of glucose uptake in normal and mildly diabetic subjects. Diabetes 19, 571-578 (1970)

3. Shen, S-W, Reaven, G.M., Farquhar, J.W.: Comparison of impedance to insulin mediated glucose uptake in normal and diabetic subjects. J. Clin. Invest. 49, 2151-2160 (1970)

4. Olefsky, J., Farquhar, J.W., Reaven, G. M.: Relationship between fasting plasma insulin level and resistance to insulinmediated glucose uptake in normal and diabetic subjects. Diabetes 22, 507-513 (1973)

5. Ginsberg, H., Olefsky, J.M., Reaven, G.M.: Further evidence that insulin resistance exists in patients with chemical diabetes. Diabetes 23, 674-678 (1974)

6. Ginsberg, H., Kimmerling, G., Olefsky, J.M., Reaven, G. M.: Demonstration of insulin resistance in untreated adult onset diabetic subjects with fasting hyperglycaemia. J. Clin. Invest. 55, 454-461 (1975)

7. Reaven, G. M., Bernstein, R., Davis, B., Olefsky, J. M.: Nonketotic diabetes mellitus: insulin deficiency or insulin resistance? Am. J. Med. 60, 80-88 (1976)

8. Kadish, A.H., Litle, R. L., Stemberg, J. C.: A new and rapid method for the determination of glucose by measurement of rate of oxygen consumption. Clin. Chem. 14, 116-131 (1968)

9. Desbuquois, B., Aurbach, G.D.: Use of polyethylene glycol to separate free and antibody bound peptide hormones in radioimmunoassays. J. Clin. Endocrinol. 33, 732-738 (1971)

10. Pupo, A. A., Mileni, J.M., Ursich, J.M., Tamaguchi, E., Vanconcellos, F. G.: Acute and late phase insulin secretion and glucose tolerance in mild alloxan diabetes in dogs. Diabetes 25, 161-166 (1976)

11. Kimmerling, G., Javorski, C., Olefsky, J.M., Reaven, G. M.: Localization of the site(s) of insulin resistance in patients with nonketotic diabetes mellitus. Diabetes 25, 673-678 (1976)

12. Hansen, A.P.: Normalization of growth hormone hyperresponse to exercise in juvenile diabetics after "normalization" of blood sugar. J. Clin. Invest. 50, 1806-1811 (1971)

13. Raskin, P., Fujita, Y., Unger, R. H.: Effect of insulin-glucose infusions on plasma glucagon levels in fasting diabetics and nondiabetics. J. Clin. Invest. 56, 1132-1138 (1975)

14. Olefsky, J.M., Reaven, G.M.: Decreased insulin binding to lymphocytes from diabetic subjects. J. Clin. Invest. 54, 1323-1328 (1974)

15. Olefsky, J.M., Reaven, G. M.: Effects of sulfonylurea therapy on insulin binding to mononuclear leukocytes of diabetic patients. Am. J. Med. 60, 89-95 (1976)

16. Galton, D. J., Wilson, J.P.D.: The effect of starvation and diabetes on glycolytic enzymes in human adipose tissue. Clin. Sci. 41, 545-553 (1971)

Received: January 7, 1977, and in revised form: April 15, 1977

Gerald M. Reaven, M. D.

Veterans Administration Hospital

3801 Miranda Avenue

Palo Alto, CA 94304

USA 